\title{
The impact of time-frequency estimation methods on the performance of wave energy converters under passive and reactive control
}

\author{
Paula B. Garcia-Rosa, Member, IEEE, John V. Ringwood, Senior Member, IEEE, \\ Olav B. Fosso, Senior Member, IEEE, and Marta Molinas, Member, IEEE
}

\begin{abstract}
A number of wave energy controllers tune the power take-off (PTO) system to the frequency of incident waves. Since real ocean waves are non-stationary by nature and not defined by a single frequency component, the PTO can be either tuned at a constant frequency characterized by the local spectrum, or continuously tuned to a representative wave frequency. In either case, a time-frequency representation of the waves is expected since the wave profile changes over time. This paper discusses about the PTO tuning problem for passive and reactive controllers, in real waves, by comparing different methods for time-varying frequency estimation: the extended Kalman filter (EKF), frequency-locked loop (FLL), and Hilbert-Huang transform (HHT). The aim is to verify the impact of such methods on the absorbed and reactive powers, and the PTO rating. It is shown that the mean estimated frequency of the EKF, and FLL, converges respectively to the mean centroid frequency, and energy frequency, of the excitation force spectrum. Moreover, the HHT mean frequency has no correlation with the spectral statistical properties. A comparison of the energy absorbed shows that up to $37 \%$ more energy is obtained with the HHT over the other estimation methods. Numerical simulations are performed with sea elevation data from the Irish coast.
\end{abstract}

Index Terms-Energy harvesting, wave power, frequency estimation, control systems.

\section{INTRODUCTION}

$\mathbf{I}$ $\mathrm{N}$ order to optimize the energy extracted from waves, a number of control algorithms for wave energy converters (WECs) tune the power take-off (PTO) system to the frequency of incident waves, see, e.g., [1]-[6]. For regular waves, the PTO tuning is well defined since the waves are characterized by a constant frequency sinusoidal motion. Nevertheless, real ocean waves are non-stationary by nature and not defined by a single frequency. In deep water, wind-generated waves can be categorized as a Gaussian stochastic process with statistical properties evaluated through the frequency and probability domains [7]. Thus, the PTO can be either tuned to a constant representative frequency of the local wave spectrum, or continuously tuned to a time-varying frequency.

P. B. Garcia-Rosa and Olav B. Fosso are with the Dept.of Electric Power Eng., Norwegian Univ. of Science and Technology (NTNU), Trondheim, Norway (emails: p.b.garcia-rosa@ieee.org, olav.fosso@ntnu.no). This work was developed while P. B. Garcia-Rosa was a visiting researcher at Dept. of Eng. Cybernetics (NTNU) with a CNPq-Brazil fellowship (grant number 201773/2015-5)

J. V. Ringwood is with the Centre for Ocean Energy Research, Maynooth University, Maynooth, Ireland (e-mail:john.ringwood@nuim.ie).

M. Molinas is with the Dept. of Eng. Cybernetics, Norwegian Univ. of Science and Technology, Trondheim, Norway (email: marta.molinas@ntnu.no).
The PTO tuning consists of adjusting the damping of the system, if a passive control method is adopted, or it may require adjustment of both the system damping and the stiffness for a reactive control method. The tuning strategies usually require knowledge of the incident wave frequency [1]-[5] or the excitation force frequency [8], [9]. Since these frequencies change with time for non-stationary signals, a time-frequency representation for the waves is expected - i.e. the frequency is a function of time.

By adopting different methods to estimate the time-varying frequency for irregular waves and real ocean waves, some studies show that continuously tuning the PTO result in greater energy absorption than tuning the PTO to a constant frequency of the wave spectrum [2], [9]. In [2], two methods have been adopted to estimate the on-line dominant wave frequency. One is based on the sliding discrete Fourier transform, while the other uses an analysis of the low-pass filtered incident wave, where wave characteristics, such as the zero-up crossing and crest-crest periods are calculated. In [9], an estimation of the instantaneous frequency of the excitation force is obtained by means of the Hilbert-Huang transform (HHT), and such information is used for tuning the PTO damping on a waveby-wave basis. Other methods have adopted the extremumseeking approach, where knowledge of the wave frequency is not needed for tuning purposes [10], [11]. In such cases, the PTO parameters are adapted on an hourly basis (according to sea states variations) rather than a wave-by-wave basis.

Furthermore, a number of control algorithms also rely on the estimation of a time-varying frequency or the energy period of the waves [6], [8], [12]-[15]. An on-line estimate of the excitation force frequency is obtained with the extended Kalman filter (EKF) in [8]. In the proposed controller, the velocity reference is set as the ratio between the excitation force and the radiation damping, which is tuned to the estimated frequency [8]. An adaptive vectorial control approach is proposed in [13], where a frequency-locked loop (FLL) is adopted to estimate the frequency of the WEC velocity.

The EKF-based method is based on a harmonic model with one variable frequency [8]. Then, the EKF tracks only a single dominant frequency. In contrast, the FLL method is based on an adaptive filter structure and an integral controller, where the selectivity of the filter and the tracking frequency dynamics can be adjusted [16]. Moreover, the instantaneous frequency, defined by the derivative of a phase function, is a time-varying parameter which identifies the location of 
the spectral peak of the signal as it varies with time [17]. However, the instantaneous frequency has physical meaning only for mono-component signals, i.e., signals with a single frequency or a narrow range of frequencies varying as a function of time [17]. In order to calculate the instantaneous frequency of multi-component signals, the HHT method [18] firstly decomposes the original signal into a number of monocomponent signals through the empirical mode decomposition (EMD). Such a decomposition has an adaptive basis and relies on the local characteristics of the signal. Thus, the EMD can extract different oscillation modes present in a wave profile.

Although previous studies, e.g., [8], [9], [13], have focused on the use of a WEC controller based on instantaneous frequency estimates, a comparison of the effectiveness of various methods to estimate the instantaneous frequency of real ocean waves has not yet been performed. This paper focuses on such a comparison. The aim is to verify the impact on the WEC performance of adopting different frequency estimation methods for tuning purposes in real ocean waves. Three methods that have recently been used for wave energy control purposes are adopted for frequency estimation: EKF, FLL, and HHT. The methods are conceptually different and fall into three diverse classes: state observer (EKF), adaptive filtering (FLL), and empirical time-frequency analysis method (HHT). The frequency estimates vary from a single dominant frequency (EKF) to the instantaneous wave-to-wave frequency of the oscillation modes present in a wave profile (HHT).

This study considers both passive and reactive controllers. For reactive control, the total power consists of a combination of active and reactive powers. Therefore, it is fundamental to individually determine these two power components to indicate the actual absorbed power, and the power that has to be supplied by the PTO during the conversion process. The WEC performance is then measured in terms of absorbed power, peak-to-average power ratio (PTO rating), and ratio of average reactive power and absorbed power. Numerical simulations with real sea elevation data from the Irish west coast are presented.

\section{MOdELING AND CONTROL OF WECS}

\section{A. Equations of Motion}

This study considers a single oscillating-body represented as a truncated vertical cylinder constrained to move in heave. With the assumption of linear hydrodynamic theory, and neglecting friction and viscous forces, the motion of the floating cylinder is described by the superposition of the wave excitation force $\left(f_{e}\right)$, radiation force $\left(f_{r}\right)$, restoring force $\left(f_{s}\right)$ and the force produced by the PTO mechanism $\left(f_{p}\right)$ :

$$
m \ddot{x}(t)=f_{e}(t)+f_{r}(t)+f_{s}(t)+f_{p}(t),
$$

where $x \in \mathbb{R}$ is the vertical position of the body, and $m \in$ $\mathbb{R}_{+}$is the body mass. The restoring force is given by $f_{s}=$ $-S x$, where $S \in \mathbb{R}_{+}$is the buoyancy stiffness. From [19], the radiation force is calculated as

$$
-f_{r}(t)=m_{r}(\infty) \ddot{x}+\int_{0}^{t} h_{r}(t-\tau) \dot{x}(\tau) d \tau,
$$

where $m_{r}(\infty) \in \mathbb{R}_{+}$is the infinite-frequency added mass coefficient, defined with the asymptotic values of the added masses at infinite frequency. The kernel of the convolution term $h_{r}(t-\tau)$ is known as the fluid memory term:

$$
h_{r}(t)=\frac{2}{\pi} \int_{0}^{\infty} B_{r}(\omega) \cos (\omega t-\tau) d \omega
$$

where $B_{r}(\omega) \in \mathbb{R}_{+}$is the radiation damping coefficient, and $\omega \in \mathbb{R}_{+}$is the wave frequency. From (1) and (2),

$$
M \ddot{x}(t)+\int_{0}^{t} h_{r}(t-\tau) \dot{x}(\tau) d \tau+S x(t)=f_{e}(t)+f_{p}(t),
$$

with $M=\left[m+m_{r}(\infty)\right]$. The excitation force, i.e., the force due to the incident waves is given by

$$
\begin{aligned}
f_{e}(t) & =\int_{-\infty}^{\infty} h_{e}(t-\tau) \zeta(\tau) d \tau, \\
h_{e}(t) & =\frac{1}{2 \pi} \int_{-\infty}^{\infty} H_{e}(\omega) e^{i \omega t} d \omega .
\end{aligned}
$$

$h_{e}$ is the inverse Fourier transform of the excitation force transfer function $H_{e}(\omega)$, which has low-pass filter characteristics for floating WECs, and $\zeta$ is the wave elevation. Notice that (6) is non-causal, since in fact, the pressure distribution is the cause of the force and not the incident waves [20].

\section{B. Passive Control}

A generic PTO mechanism, with a damper varying in time $\left(B_{p} \in \mathbb{R}_{+}\right)$, is considered for the passive control (PC). Thus,

$$
f_{p}(t)=-B_{p}(t) \dot{x}(t)
$$

For the case when $B_{p}(t)=B_{p}$, for any time $t$, and for monochromatic waves, the maximum absorption is obtained when [20]:

$$
B_{p}=\sqrt{\left(B_{r}(\omega)\right)^{2}+\left(\omega\left(m+m_{r}(\omega)\right)-S / \omega\right)^{2}},
$$

where $m_{r}(\omega) \in \mathbb{R}$ is the added mass. Equation (8) is frequency dependent, and indicates that there is an optimal damping for each frequency when the WEC is submitted to real ocean waves, or irregular waves with a mixture of frequencies.

Here, the PTO damping is continuously modified, and tuned to the excitation force frequency. From (8),

$$
B_{p}(t)=\sqrt{\left(B_{r}(\hat{\omega})\right)^{2}+\left(\hat{\omega}\left(m+m_{r}(\hat{\omega})\right)-S / \hat{\omega}\right)^{2}},
$$

where $\hat{\omega}(t)$ is the estimated time-domain frequency of the wave excitation force. In order to examine the impact of the time-varying frequency on the WEC performance, three methods are adopted for the frequency estimation: EKF, FLL, and HHT. 


\section{Reactive Control}

For reactive control (RC), the PTO force consists of a damping term and a spring term:

$$
f_{p}(t)=-B_{p}(t) \dot{x}(t)-S_{p}(t) x(t),
$$

where $S_{p} \in \mathbb{R}$ is the stiffness coefficient. For incident regular waves, if $S_{p}(t)=S_{p}$ for any time $t$, and

$$
S_{p}=\omega^{2}\left(m+m_{r}(\omega)\right)-S,
$$

then the reactive part of the total impedance

$$
\left[R_{p}+R_{r}(\omega)\right]+j \omega\left[\left(m+m_{r}(\omega)-\left(S+S_{p}\right) / \omega^{2}\right)\right]
$$

is cancelled and the velocity of the floating body is in phase with the excitation force [20]. In such a case, (8) becomes

$$
B_{p}=B_{r}(\omega)
$$

and the greatest wave energy absorption is obtained.

Following the same procedure for PC, $S_{p}$ and $B_{p}$ are tuned to the excitation force frequency. From (11) and (12),

$$
\begin{aligned}
S_{p}(t) & =\hat{\omega}^{2}(t)\left(m+m_{r}(\hat{\omega}(t))\right)-S, \\
B_{p}(t) & =B_{r}(\hat{\omega}(t)) .
\end{aligned}
$$

Notice that, for practical application studies, the physical limits of the body excursion and the PTO should be taken into account. Here, a PTO force constraint is implemented as a saturation. However, this is only a theoretical approach, since the body motion is also a function of the excitation force, which is an external force that cannot be manipulated. The implementation of saturation on the force signals of a real WEC is not physically possible.

\section{Energy and Power}

The mean power and energy absorbed by the WEC over a time range $T$ are, respectively,

$$
\bar{P}=\frac{E}{T}, \quad \text { and } \quad E=-\int_{0}^{T} B_{p}(t) \dot{x}^{2}(t) d t .
$$

Notice that, for the reactive control, the delivered power has two components: the absorbed power (or active power, that is the power delivered to the damping $B_{p}$ ), and the reactive power (the power delivered to the spring $S_{p}$ ) [20]. The mean reactive power, and reactive energy, are respectively,

$$
\bar{P}_{r}=\frac{E_{r}}{T}, \quad \text { and } \quad E_{r}=-\int_{0}^{T} S_{p}(t) x(t) \dot{x}(t) d t .
$$

Since the spring term in the PTO force (10) and the body velocity can have opposite signs in (16), the PTO has to return energy for some parts of the wave cycle. Then, the PTO system should be able to implement bidirectional power flow for RC.

Here, the WEC performance in terms of mean absorbed power $(\bar{P})$ is measured by the capture width ratio [21],

$$
\mathrm{CWR}=\frac{\bar{P}}{2 r P_{\zeta}},
$$

where $r$ is the cylinder radius and $P_{\zeta}$ is the transported wave power per unit width of the wave front. In deep water [20],

$$
P_{\zeta}=\frac{\rho g}{2} \int_{0}^{\infty} \frac{S_{\zeta}(\omega)}{\omega} d \omega,
$$

where $S_{\zeta}$ is the wave spectrum, $\rho$ is the sea water density, and $g$ is the gravitational acceleration.

\section{ESTIMATION OF THE WAVE EXCITATION FORCE FREQUENCY}

\section{A. Extended Kalman Filter}

In order to estimate the frequency by means of the EKF, we assume a harmonic model with a single time-varying frequency component and amplitude, as proposed in [22]. Thus, $f_{e}(t)$ can be expressed in discrete-time $\left(t=k T_{s}\right)$ as:

$$
f_{e}[k]=A[k] \cos \left(\omega[k] k T_{s}+\varphi[k]\right)+\eta[k],
$$

where $A[k]$ is the excitation force amplitude, $\varphi[k]$ is the phase, and $T_{s}$ is the sampling time. Following the cyclical structural model from [23], but with a slowly time-varying frequency:

$$
\begin{aligned}
{\left[\begin{array}{c}
\psi[k+1] \\
\psi^{*}[k+1]
\end{array}\right] } & =\left[\begin{array}{ll}
\cos \left(\omega[k] k T_{s}\right) & -\sin \left(\omega[k] k T_{s}\right) \\
\sin \left(\omega[k] k T_{s}\right) & \cos \left(\omega[k] k T_{s}\right)
\end{array}\right]\left[\begin{array}{c}
\psi[k] \\
\psi^{*}[k]
\end{array}\right]+\left[\begin{array}{c}
\varrho[k] \\
\varrho^{*}[k]
\end{array}\right] \\
f_{e}[k] & =\psi[k]+\eta[k],
\end{aligned}
$$

where $\left\{\psi[k], \psi^{*}[k]\right\} \in \mathbb{R}$ are in-phase and quadrature signals, and $\left\{\varrho[k], \varrho^{*}[k]\right\} \in \mathbb{R}$ are assumed to be zero-mean uncorrelated noise processes with same variance. Such assumptions are imposed here to reduce the complexity [23].

Thus, by defining a state vector as $v=\left[\begin{array}{lll}\psi & \psi^{*} & \omega\end{array}\right]^{T}$, and a noise vector as $\varpi=\left[\begin{array}{lll}\varrho & \varrho^{*} & \varsigma\end{array}\right]^{T}$, (20) can be written in a recursive non-linear state space form as:

$$
\begin{aligned}
v[k+1] & =f(v[k])+\varpi[k] \\
f_{e}[k] & =h(v[k])+\eta[k]
\end{aligned}
$$

where $\varpi[k] \in \mathbb{R}^{3}$ and $\eta[k] \in \mathbb{R}$ are zero-mean, independent noise processes with covariance matrices defined, respectively, as $E\left[\varpi \varpi^{T}\right]=Q$ and $E\left[\eta \eta^{T}\right]=R$. Here, $Q=\operatorname{diag}\left(q_{1}, q_{1}, q_{2}\right)$, and $R$ is a scalar, thus, $R \equiv r$. Functions $f(\cdot)$ and $h(\cdot)$ are,

$$
f(v[k])=\left[\begin{array}{ccc}
\cos \left(\omega[k] T_{s} k\right) & -\sin \left(\omega[k] T_{s} k\right) & 0 \\
\sin \left(\omega[k] T_{s} k\right) & \cos \left(\omega[k] T_{s} k\right) & 0 \\
0 & 0 & 1
\end{array}\right] v[k],
$$

and $h(v[k])=\left[\begin{array}{lll}1 & 0 & 0\end{array}\right] v[k]$, respectively [22].

The EKF obtains an estimate of the state vector $v[k]$ based on observations of $f_{e}[k]$, and on the first-order linearization of model (21) around the last state estimate. The EKF algorithm is summarized in Table I, where $I$ is the identity matrix of order $3, J_{f}$ and $J_{h}$ are the Jacobian matrices of $f($.$) and h($.$) ,$ denoted respectively by $J_{f}[k]=\left.\nabla f\right|_{\hat{v}[k \mid k]}$ and $J_{h}[k+1]=$ $\left.\nabla h\right|_{\hat{v}[k+1 \mid k]}$. Once an estimate of the state vector $\hat{v}[k \mid k]$ is available from the EKF (Table I), the amplitude and frequency of the wave excitation force are, respectively, obtained as:

$$
\begin{aligned}
\hat{A}_{\mathrm{EKF}}[k \mid k] & =\sqrt{\hat{\psi}[k \mid k]^{2}+\hat{\psi}^{*}[k \mid k]^{2}}, \\
\hat{\omega}_{\mathrm{EKF}}[k \mid k] & =\hat{\omega}[k \mid k] .
\end{aligned}
$$


TABLE I

EKF ALGORITHM.

\begin{tabular}{|l|}
\hline Prediction step: \\
$\hat{v}[k+1 \mid k]=f(\hat{v}[k \mid k])$ \\
$P[k+1 \mid k]=J_{f}[k] P[k \mid k] J_{f}[k]^{T}+Q[k]$ \\
\hline Innovation step: \\
$\hat{v}[k+1 \mid k+1]=\hat{v}[k+1 \mid k]+K[k+1]\left(f_{e}[k+1]-h(\hat{v}[k+1 \mid k])\right)$ \\
$K[k+1]=P[k+1 \mid k] J_{h}[k+1]^{T}\left(J_{h}[k+1] P[k+1 \mid k] J_{h}[k+1]^{T}+R[k+1]\right)^{-1}$ \\
$P[k+1 \mid k+1]=\left(I-K[k+1] J_{h}[k+1]\right) P[k+1 \mid k]$ \\
\hline
\end{tabular}

Theoretical guidelines for the design of stable EKF frequency trackers are given in [24]. The covariance matrices should be defined as $Q \geq 0$ and $R>0$. The choice of $R$ and $Q$ affects the stability properties and the rate of response of the EKF frequency tracker [24]. A high value of $Q$ allows the EKF to track signals with high rate of change of the frequency. However, the filter will be oversensitive to noise, the estimates will fluctuate widely around the true value of the frequency, and the results might become inaccurate. Thus, it is important to design a sufficiently conservative filter [24]. Moreover, a high value of $R$ reduces the sensitivity of the filter to noise, but it decreases the speed response of the filter.

\section{B. Frequency-Locked Loop}

An adaptive filter is implemented by means of a secondorder generalized integrator (SOGI), where the FLL estimates the frequency of the input signal. Such a scheme is termed SOGI-FLL, and has been proposed for grid synchronization of power converters [16]. Figure 1 illustrates the block diagram of the SOGI-FLL, consisting of the SOGI-QSG (SOGI quadrature signal generator) and the FLL. The method was first adopted, within the wave energy control context, to estimate the frequency of the WEC velocity [13]. Here, the method is adopted for estimating the wave excitation force frequency.

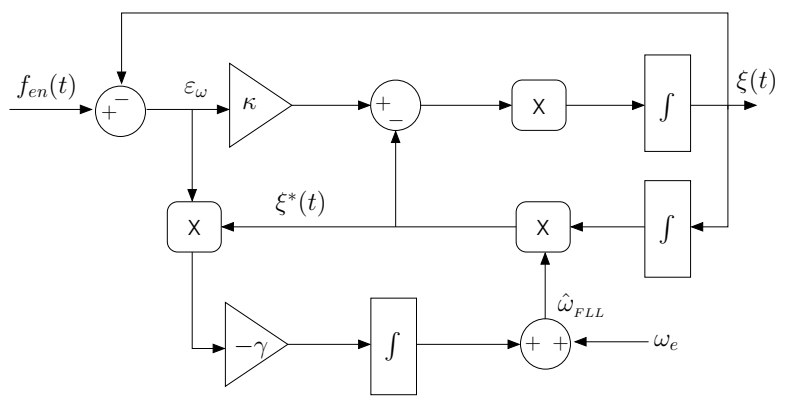

Fig. 1. Frequency estimation by the SOGI-FLL method.

The SOGI-QSG acts as an adaptive bandpass filter with two in-quadrature output signals $\left(\xi, \xi^{*}\right)$, where $\xi^{*}$ lags $\xi$ by $90^{\circ}$. The bandwidth of the filter is exclusively set by the gain $\kappa$ [16]. The FLL is responsible for estimating the frequency of the input signal. Notice that in the nonlinear frequency adaptation loop, $\varepsilon=\varepsilon_{\omega} \xi^{*}$ can be interpreted as a frequency error variable, and the parameter $\gamma$ represents the gain of the integral controller [16]. The selectivity of the adaptive bandpass filter and the tracking frequency dynamics are respectively determined by the tuning parameters $\kappa$ and $\gamma$.
From Fig. 1, the state-space equations of the SOGI-FLL are

$$
\begin{aligned}
{\left[\begin{array}{c}
\dot{\xi} \\
\dot{\nu}
\end{array}\right] } & =\left[\begin{array}{cc}
-\kappa \hat{\omega}_{\mathrm{FLL}} & -\hat{\omega}_{\mathrm{FLL}}^{2} \\
0 & 1
\end{array}\right]\left[\begin{array}{l}
\xi \\
\nu
\end{array}\right]+\left[\begin{array}{c}
\kappa \hat{\omega}_{\mathrm{FLL}} \\
0
\end{array}\right] f_{e n} \\
{\left[\begin{array}{c}
\xi \\
\xi^{*}
\end{array}\right] } & =\left[\begin{array}{cc}
1 & 0 \\
0 & \hat{\omega}_{\mathrm{FLL}}
\end{array}\right]\left[\begin{array}{l}
\xi \\
\nu
\end{array}\right] \\
\dot{\hat{\omega}}_{\mathrm{FLL}} & =-\gamma\left(f_{e n}-\xi\right) \xi^{*} \hat{\omega}_{\mathrm{FLL}},
\end{aligned}
$$

where $(\xi, \nu)$ and $\left(\xi, \xi^{*}\right)$ are, respectively, the state and output vectors of the SOGI, and $f_{e n}$ is the normalized excitation force. The FLL state equation is represented by (25).

\section{Hilbert-Huang Transform}

In the HHT method, the wave excitation force $f_{e}(t)$ is firstly decomposed into $N$ mono-component signals (IMFs) by the EMD. Then, the instantaneous frequency of the dominant IMF is adopted for tuning purposes [9]. Figure 2 illustrates the block diagram of the frequency estimation by this method.

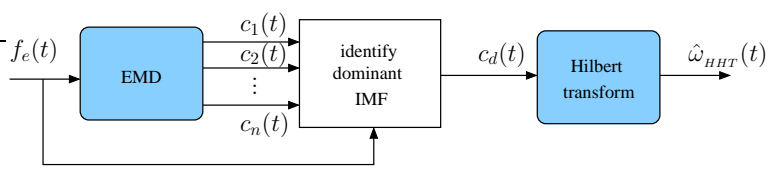

$\square$ HHT method

Fig. 2. Frequency estimation by the HHT method.

The EMD identifies local maxima and minima of $f_{e}(t)$, and calculates upper and lower envelopes for such extrema using cubic splines. The mean values of the envelopes are used to decompose the original signal into frequency components in a sequence from the highest frequency to the lowest one. The EMD procedure is summarized in Table II.

\begin{tabular}{|c|}
\hline Step 0: Set $i=1 ; r(t)=f_{e}(t)$; \\
\hline Step 1: Identify the local maxima and minima in $r(t)$; \\
\hline $\begin{array}{l}\text { Step 2: Calculate the upper envelope defined by the maxima, } \\
\text { and the lower envelope defined by the minima; }\end{array}$ \\
\hline Step 3: Calculate the mean envelope $m(t)$; \\
\hline Step 4: Set $h(t)=r(t)-m(t)$ \\
\hline $\begin{array}{l}\text { Step 5: If } h(t) \text { is an IMF, go to next step. Otherwise, set } r(t)=h(t) \\
\text { and go back to step 1; }\end{array}$ \\
\hline Step 6: Set $c_{i}(t)=h(t) ; r(t)=r(t)-c_{i}(t)$ \\
\hline $\begin{array}{l}\text { Step 7: If } i=N \text {, define the IMF components as } c_{1}(t), \ldots, c_{N}(t) \text {, } \\
\text { and the residue as } r(t) \text {. Otherwise, set } i=i+1 \text { and } \\
\text { go back to step } 1 .\end{array}$ \\
\hline
\end{tabular}

TABLE II

EMD ALGORITHM.

Then, the wave excitation force can be expressed as

$$
f_{e}(t)=\sum_{i=1}^{N} c_{i}(t)+r(t),
$$

where $N$ is the total number of IMFs, which is defined here as $\log _{2} N_{s}-1$ [25], $N_{s}$ is the data length, and $r(t)$ is the residue.

The dominant IMF is identified through the comparison of the energy of the IMF signals $\left(E_{c_{i}}\right)$ with the energy of the excitation force signal $\left(E_{f_{e}}\right)$,

$$
E_{c_{i}}=\int_{0}^{T}\left|c_{i}(t)\right|^{2} d t, \quad E_{f_{e}}=\int_{0}^{T}\left|f_{e}(t)\right|^{2} d t,
$$


where $c_{i}(t)$ is the $i$-th IMF component. The dominant component $c_{d}(t)$ is the IMF with the highest $E_{c_{i}} / E_{f_{e}}$ ratio.

Finally, the Hilbert transform (HT) is applied to $c_{d}(t)$ [18]:

$$
v_{d}(t)=\frac{1}{\pi} P \int_{-\infty}^{\infty} \frac{c_{d}(\tau)}{t-\tau} d \tau
$$

where $P$ indicates the Cauchy principal value. Then, the dominant IMF is represented as an analytic signal,

$$
z_{d}(t)=c_{d}(t)+j v_{d}(t),
$$

with amplitude $\hat{A}_{\text {ннт }}$, phase $\hat{\phi}_{\text {ннт }}$, and instantaneous frequency $\hat{\omega}_{\text {ннт }}$, respectively estimated as

$$
\begin{gathered}
\hat{A}_{\text {ннт }}(t)=\sqrt{c_{d}^{2}(t)+v_{d}^{2}(t)}, \hat{\phi}_{\text {ннт }}(t)=\arctan \left(\frac{v_{d}(t)}{c_{d}(t)}\right), \\
\hat{\omega}_{\text {ннт }}(t)=\dot{\phi}_{d}(t) .
\end{gathered}
$$

\section{Simulation RESULtS}

\section{A. Hydrodynamic parameters}

The same heaving cylinder adopted in [9] is considered here. The cylinder has a radius of $r=5 \mathrm{~m}$, draught $d=4 \mathrm{~m}$, mass $m=3.2 \times 10^{5} \mathrm{~kg}$ and resonance frequency $1.2 \mathrm{rad} / \mathrm{s}$. The hydrodynamic coefficients were computed using the boundary element solver WAMIT [26]. The added mass, radiation damping coefficients, and the frequency response of the excitation force are shown in [9].

\section{B. Real sea elevation data}

The wave data was collected in 2010 from a data buoy in the Belmullet wave energy test site, off the west coast of Ireland. The wave data, provided by the Irish Marine Institute, consists of wave elevation records of 30 minutes sampled at $1.28 \mathrm{~Hz}$.

Six wave elevation records (referred as sea states S1-S6), with different spectral distribution, were selected for our study. Figure 3 illustrates the wave spectra of the sea states, and Table III shows the significant wave height $\left(H_{s}\right)$, the peak frequency $\left(\omega_{p}\right)$, the energy frequency $\left(\omega_{e}\right)$, the mean centroid frequency $\left(\omega_{1}\right)$, and the mean zero-crossing frequency $\left(\omega_{2}\right)$ of the spectra. The statistical parameters are calculated as: $H_{s}=4 \sqrt{m_{0}}, \omega_{e}=m_{0} / m_{-1}, \omega_{1}=m_{1} / m_{0}, \omega_{2}=\sqrt{m_{2} / m_{0}}$, where $m_{n}=\int_{0}^{\infty} \omega^{n} S(\omega) d \omega$ is the spectral moment of order $n$. $\omega_{p}$ is the frequency at which the wave spectrum is maximum.

Figure 4 shows the spectral density of the excitation force for the selected sea states. Some of the high frequency waves are filtered out by the transfer function $H_{e}(\omega)$, as can be noted from Figure 3. The filtering characteristics are defined by the shape of the floating body, so that the excitation force spectra are characteristic of the cylinder adopted in this study.

\section{Time-frequency estimation by EKF, FLL and HHT}

1) Superposition of two regular waves: In order to illustrate how the estimated excitation force frequency differs according to the method adopted, firstly we consider a simple incident wave defined as the superposition of two regular waves: $\zeta(t)=2 \cos (2 \pi / 6 t)+\cos (2 \pi / 8 t)$. The energy frequency and
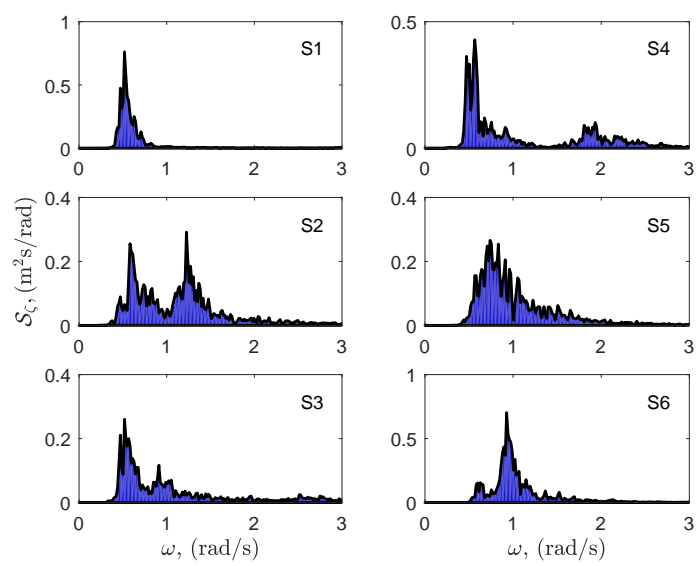

Fig. 3. Wave spectra of real wave data from Belmullet.

TABLE III

SignificANT WAVE HEIGHT $H_{s}(\mathrm{M})$, PEAK FREQUENCY $\omega_{p}$, ENERGY FREQUENCY $\omega_{e}$, MEAN CENTROID FREQUENCY $\omega_{1}$, AND MEAN ZERO-CROSSING FREQUENCY $\omega_{2}$ OF THE SELECTED SEA STATES. FREQUENCIES IN RAD/S.

\begin{tabular}{|c|c|c|c|c|c|c|}
\hline & S1 & S2 & S3 & S4 & S5 & S6 \\
\hline$H_{s}$ & 1.26 & 1.43 & 1.18 & 1.39 & 1.42 & 1.62 \\
\hline$\omega_{p}$ & 0.52 & 1.22 & 0.52 & 0.57 & 0.74 & 0.93 \\
\hline$\omega_{e}$ & 0.59 & 0.94 & 0.80 & 0.80 & 0.90 & 0.97 \\
\hline$\omega_{1}$ & 0.70 & 1.18 & 1.13 & 1.18 & 1.06 & 1.08 \\
\hline$\omega_{2}$ & 0.88 & 1.33 & 1.38 & 1.42 & 1.18 & 1.16 \\
\hline
\end{tabular}
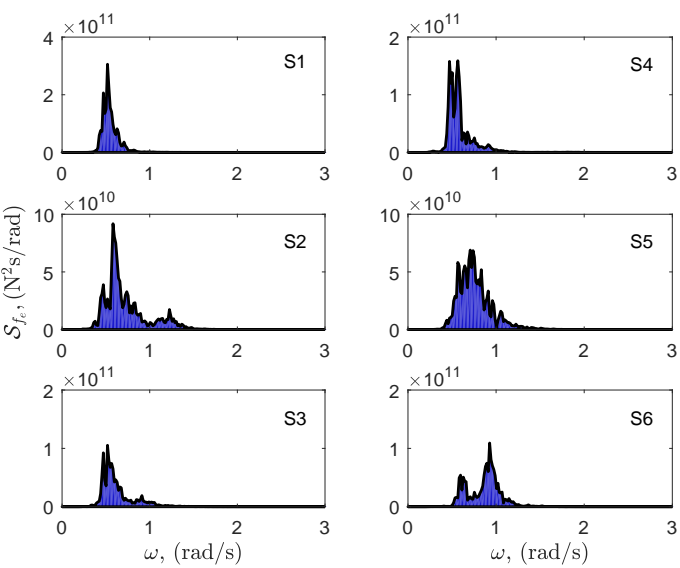

Fig. 4. Excitation force spectra for sea states S1-S6.

the mean centroid frequency of the excitation force spectra are, respectively, $\omega_{e, f_{e}}=0.94 \mathrm{rad} / \mathrm{s}$ and $\omega_{1, f_{e}}=0.96 \mathrm{rad} / \mathrm{s}$.

Figure 5 illustrates the excitation force frequency estimated by the EKF, FLL, and HT. The Hilbert spectrum shows that the instantaneous frequency varies from about 0.92 to 1.4 $\mathrm{rad} / \mathrm{s}$, with the highest energy content (highest amplitude) in the lowest frequency. In order to ensure accurate frequency estimates by the EKF, $q_{2}$ is set to a low value. By setting $r$ to $10 \operatorname{var}\left[f_{e}\right], q_{1}=r$, and $q_{2}=10^{-5}$, the EKF frequency is nearly constant. The EKF tracks a single frequency $(0.96$ $\mathrm{rad} / \mathrm{s}$ ) which represents the mean centroid frequency of the 
excitation force spectrum, and the mean frequency estimated by the FLL $(0.93 \mathrm{rad} / \mathrm{s})$ is close to the energy frequency of the spectrum. The frequency estimated by the EKF is nearly constant, while the tracking frequency dynamics of the FLL depends mainly on the selection of the parameters $\kappa$ and $\gamma$. To ensure high frequency selectivity, and accurate direct and quadrature components within frequency range 0.6 to $1 \mathrm{rad} / \mathrm{s}$, $\kappa$ is set to $\sqrt{2}$ and $\gamma=0.16$, as discussed in [13].
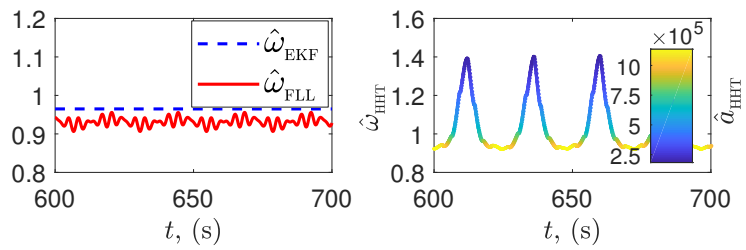

Fig. 5. Frequency estimated ( $\mathrm{rad} / \mathrm{s})$ by the EKF, FLL, and HT for the wave $\zeta(t)=2 \cos (2 \pi / 6 t)+\cos (2 \pi / 8 t)$. The plot on the right is the Hilbert spectrum.

2) Sea Elevation Data: Table IV shows the energy and mean centroid frequencies of the excitation force spectra, and the mean frequencies estimated by the studied methods for sea states S1 to S6. It can be noted that the mean values of the frequencies estimated by the EKF converge to the mean centroid frequency of the spectra, while the mean frequency values of the FLL converge to the energy frequency of the spectra. However, the mean values estimated by the HHT have no correlation with the statistical parameters obtained from the spectra. As has been remarked by Huang et al. [27], frequency in the Hilbert spectrum has a different meaning from Fourier spectral analysis. In Fourier spectral analysis, the existence of energy at a frequency means that a component of a sine (or a cosine) wave persisted through the entire time range of the data, whereas in the Hilbert spectrum the wave representation is local and the exact time of such oscillation is given [27].

TABLE IV

ENERGY FREQUENCY, MEAN CENTROID FREQUENCY, MEAN ZERO-CROSSING FREQUENCY OF THE EXCITATION FORCE SPECTRA, AND MEAN VALUES OF THE ESTIMATED FREQUENCIES $\left(\bar{\omega}_{\text {EKF }}, \bar{\omega}_{\mathrm{FLL}}, \bar{\omega}_{\mathrm{HHT}}\right)$. FREQUENCIES IN RAD/S.

\begin{tabular}{|c|c|c|c|c|c|c|}
\hline & S1 & S2 & S3 & S4 & S5 & S6 \\
\hline$\omega_{e, f_{e}}$ & 0.536 & 0.658 & 0.587 & 0.561 & 0.718 & 0.821 \\
\hline$\omega_{1, f_{e}}$ & 0.550 & 0.728 & 0.629 & 0.592 & 0.760 & 0.865 \\
\hline$\omega_{2, f_{e}}$ & 0.560 & 0.771 & 0.658 & 0.614 & 0.785 & 0.885 \\
\hline \hline $\bar{\omega}_{\text {EKF }}$ & 0.554 & 0.733 & 0.634 & 0.592 & 0.768 & 0.874 \\
\hline $\bar{\omega}_{\text {FLL }}$ & 0.532 & 0.629 & 0.565 & 0.547 & 0.695 & 0.804 \\
\hline $\bar{\omega}_{\text {HHT }}$ & 0.623 & 1.009 & 0.799 & 0.732 & 0.900 & 0.967 \\
\hline
\end{tabular}

The estimated frequencies for sea states $\mathrm{S} 1$ and $\mathrm{S} 2$ are illustrated in Figure 6. From the Hilbert spectrum of the first IMF, it can be noted that the frequency ranges $0.5-0.6 \mathrm{rad} / \mathrm{s}$, and $0.6-0.8 \mathrm{rad} / \mathrm{s}$, have the highest energy content, respectively, for S1 and S2. Such frequency values coincide with the estimates from the EKF and FLL, but the HHT method identifies the time at which such oscillations occur. Notice that the HHT analysis returns nine IMFs, and the first IMF is the dominant component in all studied cases, as shown in [9].
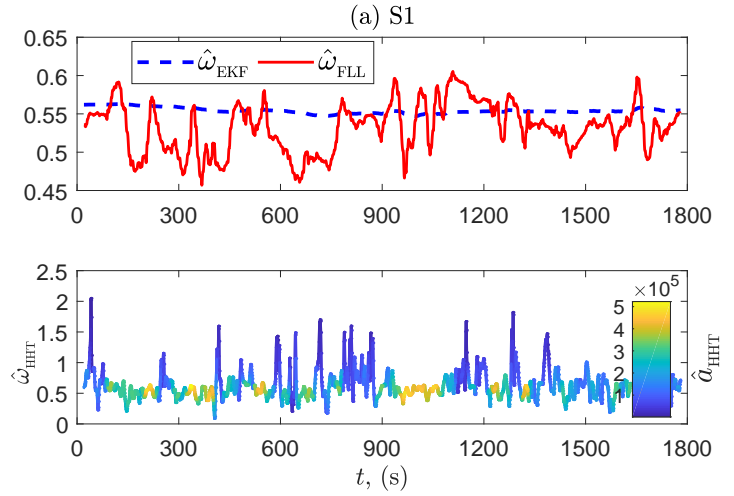

(b) S2
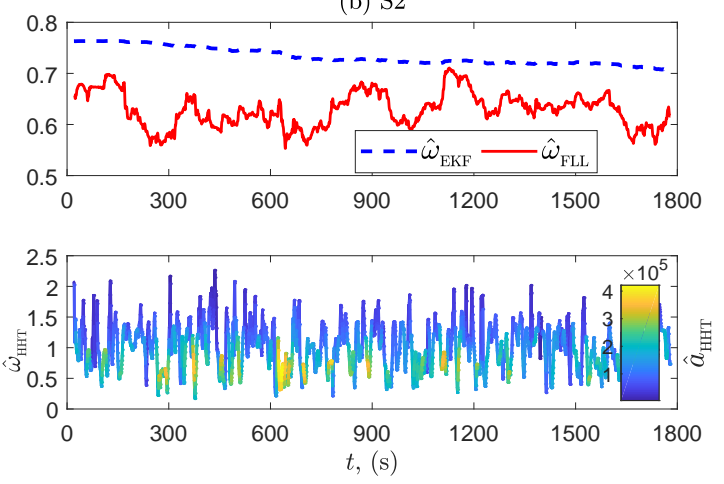

Fig. 6. Frequency estimated (rad/s) by the EKF, FLL, and HHT for (a) S1 and (b) S2. The plots in the bottom represent the Hilbert spectrum of IMF 1.

\section{Effect of the estimated frequency on the control strategy}

In order to limit the body excursions to $2.5 \mathrm{~m}$ for the studied cases, the PTO force of the PC (7) and each term of the RC in (10) is limited to $\pm 500 \mathrm{kN}$.

1) Passive Control: The performance of the WEC is illustrated in Figures 7 and 8, for the cases when the PC strategy adopts the EKF, FLL or HHT methods to estimate the excitation force frequency for sea states S1 to S6 (section IV-C2).

For all the studied cases, tuning the damping with frequency estimates from the HHT gives greater energy capture than tuning with EKF and FLL. The highest improvement is of a factor of 1.27 when HHT is compared to EKF, or 1.37 when compared to FLL, for sea state S2. Moreover, the lowest differences in the CWR is obtained for S1. Such behaviour can be explained by the different energy spectral distributions of both sea states. S1 is characterized by a narrowband spectrum with a single dominant swell (low frequency waves generated in other locations), with the energy concentrated in a narrow band of frequencies. However, S2 is characterized by a twopeak spectrum with mixed wind-sea (high frequency waves generated by the local wind) and swell conditions, with the energy spread over a wider band of frequencies than S1. In such a case, a method that calculates the wave-to-wave frequency is more beneficial for PC than a method that gives a dominant sea state frequency. Nevertheless, the PTO rating and the maximum PTO required for the HHT frequencies are also higher than for the EKF and FLL, especially for S2 (Fig. 8).

2) Reactive Control: Figures 9 and 10 illustrate the performance of the WEC, for the cases when the reactive control 


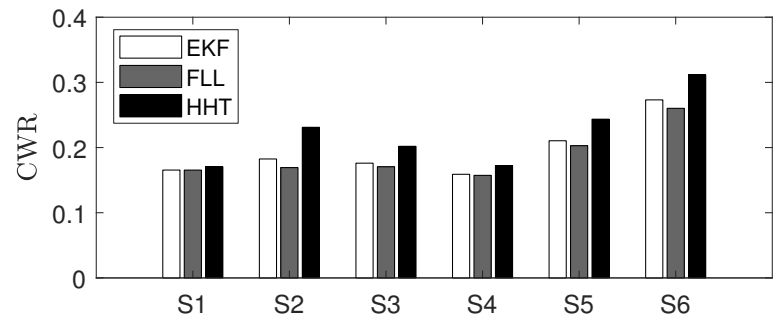

Fig. 7. CWR under PC tuned at frequencies from the EKF, FLL and HHT.

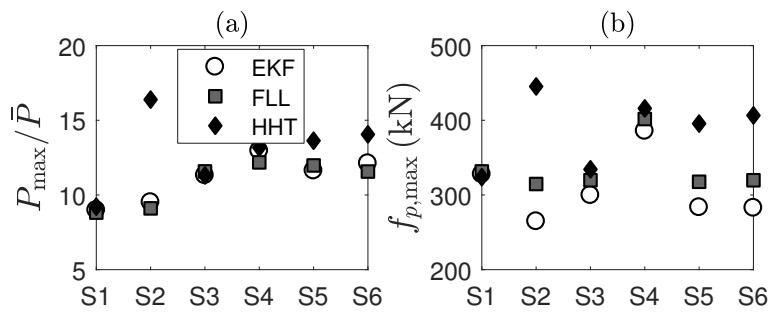

Fig. 8. WEC performance under PC (a) Peak-to-average power ratio (b) Maximum PTO force required.

strategy adopts the EKF, FLL or HHT methods to estimate the wave excitation force frequency. For the unconstrained cases, the CWR is very large, in most situations, and the HHT obtains an energy improvement of up to 2.64 over the EKF, or 1.67 over the FLL. However, the body motion ranges from -10 to $10 \mathrm{~m}$, which is practically impossible for a WEC with a draught of $4 \mathrm{~m}$. Moreover, the PTO rating for the HHT approaches a factor of 50 and the ratio of average reactive power and absorbed power is almost $60 \%$ in some cases (Fig. 10.a). Such values would require oversized PTO equipment, which would not be a rational economic choice.

For the constrained cases, the improvement obtained from HHT over EKF or FLL is lower, but represents a more realistic scenario: an average energy improvement of 1.13 is obtained when the HHT is compared to the EKF, or 1.23 when compared to the FLL. Although the PTO rating varies from a factor of 7 to 10 for all the methods, the ratio of average reactive power and absorbed power are much higher for the HHT, reaching almost 14\% (Fig. 10.b) for the studied cases.

In order to illustrate the effect of the frequency estimates on the variables of the system, for the constrained RC, Figure 11 shows samples of time-series simulation, and Figure 12 shows the absorbed and the reactive energy over a 30-min simulation interval for sea states S1-S2. It can be noted that, for sea state $\mathrm{S} 2$, the reactive energy required for the RC, tuned with HHT frequency estimates is much higher than the FLL or EKF cases. In such a case, the HHT reactive power represents about $4.2 \%$ of the total power, whereas the FLL reactive power is about $0.7 \%$. Nevertheless, the absorbed power is $18 \%$ greater with the HHT than with the FLL.

\section{E. Discussion}

In the EKF, a sinusoidal extrapolation method is used to model the excitation force as a monochromatic harmonic

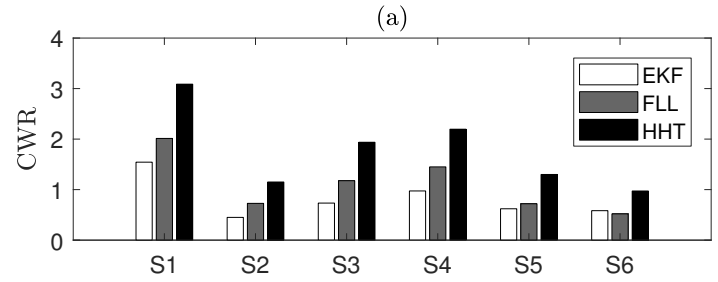

(b)

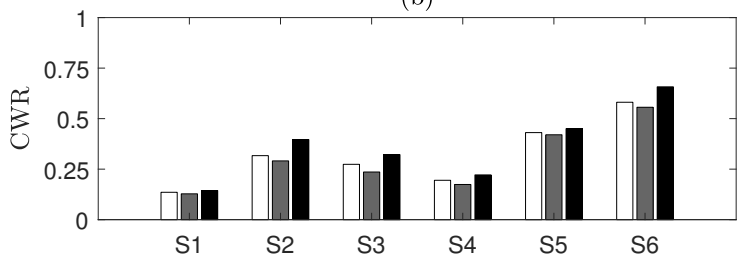

Fig. 9. CWR under RC tuned at frequencies from the EKF, FLL and HHT (a) Unconstrained case (b) Constrained case.
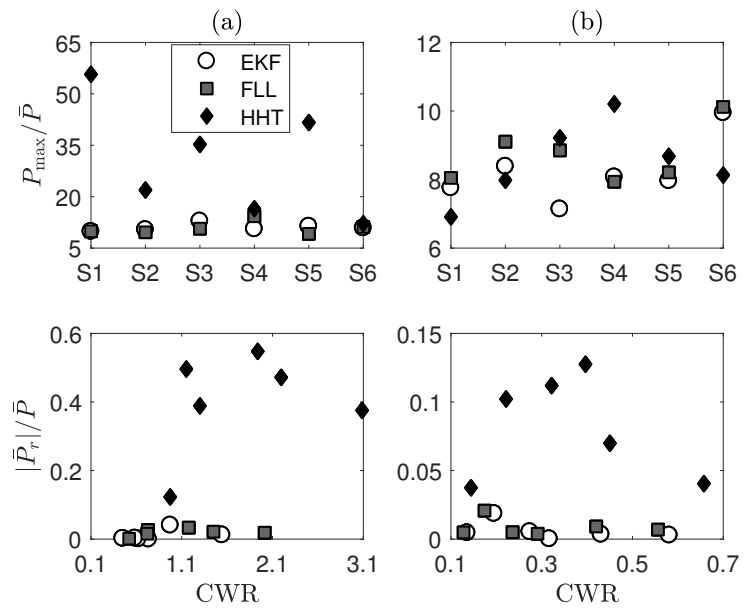

Fig. 10. WEC performance under RC: Peak-to-average power ratio (top) and ratio of average reactive power and absorbed power as a function of CWR (bottom) (a) Unconstrained case (b) Constrained case.

process, with varying amplitude and frequency. Thus, the EKF follows a single dominant frequency. Simulation results have shown that the estimated EKF frequency tracks the mean centroid frequency of the excitation force spectrum. The performance of the SOGI-FLL depends mainly on the appropriate selection of two design parameters: $\kappa$ and $\gamma$. Such parameters define the bandwidth of the adaptive filter and the FLL tracking frequency dynamics [16]. For the FLL, it has been shown that the mean estimated frequency converges to the energy frequency of the excitation force spectrum.

The HHT calculates the instantaneous frequency of the excitation force by decomposing the signal into a number of IMF components. Here, we have chosen only the IMF with the highest energy content. This component has a wider bandwidth than the estimates from the EKF or FLL, and the CWR of the WEC is greater when the HHT frequency estimates are used for PC and RC. Even with a low energy content, the resonance frequency of the WEC is within the HHT estimates, which can also explain why tuning the controllers with these estimates result in the greatest energy absorption. 
(a) S1
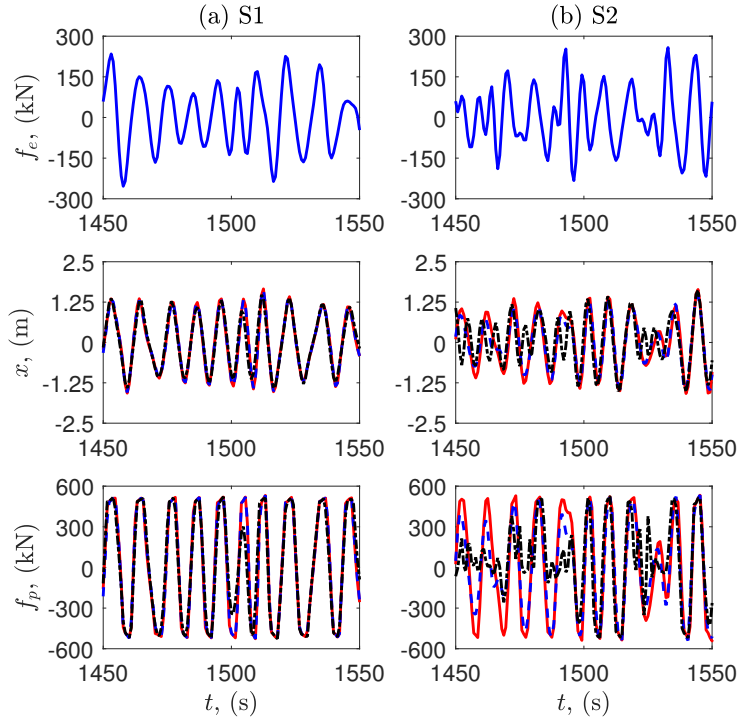

Fig. 11. Time-series of the excitation force, position, and PTO force for the constrained RC tuned with the EKF (dashed blue line), the FLL (solid red line) and the HHT (dashed dotted black line) (a) S1; (b) S2.
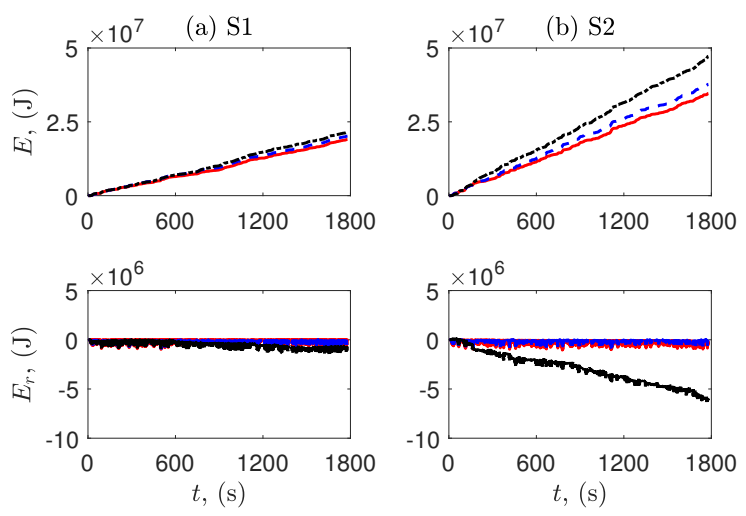

Fig. 12. Energy absorbed (top) and reactive energy (bottom) over a 30-min simulation for the constrained RC tuned with the EKF (dashed blue line), the FLL (solid red line) and the HHT (dashed dotted black line) (a)S1; (b) S2.

As expected, the PTO rating for PC is higher with the HHT than with the EKF, or FLL, in most of the cases. However, for the constrained RC, the greatest PTO rating does not indicate the greatest energy absorption. The average value of the PTO rating is about the same for all methods. Still, the greatest energy absorption, obtained with the HHT, also requires the greatest amount of reactive energy.

In both control strategies (PC and $\mathrm{RC}$ ), the control signal (PTO force) is parametrized as a function of the system states (position and velocity), and requires the knowledge of the wave excitation force. In practice, position sensors or accelerometers can be used to estimate the states, and the actuator and other related sensors will depend on the specific type of PTO system. The excitation force can be determined by using measurement of the wave elevation at some distance from the floating body, or by using an array of pressure transducers placed, e.g., on the wet surface of the body [20].

Here we have assumed the wave excitation force is known completely over the simulation interval. Both the EKF and FLL methods give an online estimate of the frequency, provided that an estimate of the excitation force is available. In the HHT method, we have adopted an off-line EMD algorithm, but a few implementation studies on the HHT have proposed real-time EMD algorithms, see, e.g., [28].

Table $\mathrm{V}$ shows the mean computational speed required to estimate the frequency of each sea state over the simulation interval $(30 \mathrm{~min})$. The HHT and the EKF required about

TABLE V

MEAN COMPUTATIONAL SPEEd OF THE METHOdS OVER 30-MIN SIMULATION INTERVAL.

\begin{tabular}{|c|c|c|}
\hline EKF & FLL & HHT \\
\hline $1.34 \mathrm{~s}$ & $0.28 \mathrm{~s}$ & $1.29 \mathrm{~s}$ \\
\hline
\end{tabular}

the same time to perform the calculations, while the FLL was the fastest method. (Computer hardware used to run the simulations: Intel(R) Core(TM) i7-7600U CPU @2.80GHz).

\section{CONCLuSion}

This paper has shown how different frequency estimation methods, used for controller tuning purposes, impact the energy absorbed by the WEC, the PTO rating and the required reactive power during the conversion process. The effect of the estimation methods on the WEC performance depend on the control strategy employed, the PTO system constraints, and the local wave spectrum.

For a control strategy that relies on the information of a dominant frequency component, such as the schemes in [8] and [14], the EKF or FLL should be adopted. The mean frequency estimated by such methods converges, respectively, to the mean centroid frequency or the energy frequency of the spectrum. Moreover, if the sea state is characterized by a narrowband spectrum, the benefit of adopting a method that estimates the wave-to-wave frequency is relatively small.

By adopting the HHT method, an average improvement in the energy absorbed of about $18 \%$ is obtained over the EKF and FLL methods, for the constrained reactive control strategy. For passive control, an average improvement of $16 \%$ is also obtained for the HHT. The greatest improvements of the HHT over the other methods are obtained for wideband spectra. In contrast to the EKF and FLL methods, where the bandwidth is narrow and the frequency estimates oscillate around a dominant frequency component, the HHT frequency estimates cover a wider range, and the location of the dominant frequency component is identified.

In this study, the first IMF component is adopted for the HHT approach. The frequency bandwidth of this IMF component could be narrowed by applying techniques that deal with mode mixing in EMD. Such an approach will be explored in future studies.

\section{ACKNOWLEDGMENT}

The authors would like to thank the Irish Marine Institute for providing the wave data from the Belmullet test site. 


\section{REFERENCES}

[1] G. Duclos, A. Babarit, and A. Clément, "Optimizing the power takeoff of a wave energy converter with regard to the wave climate," J. of Offshore Mech. and Arctic Eng., vol. 128, no. 1, pp. 56-64, 2006.

[2] H. Yavuz, T. J. Stallard, A. P. McCabe, and G. A. Aggidis, "Time series analysis-based adaptive tuning techniques for a heaving wave energy converter in irregular seas," Proc. of the Inst. of Mech. Engineers, Part A: Journal of Power and Energy, vol. 221, no. 1, pp. 77-90, 2007.

[3] U. A. Korde and R. C. Ertekin, "Wave energy conversion by controlled floating and submerged cylindrical buoys," Journal of Ocean Engineering and Marine Energy, vol. 1, no. 3, pp. 255-272, 2015.

[4] C. J. Cargo, A. J. Hillis, and A. R. Plummer, "Strategies for active tuning of wave energy converter hydraulic power take-off mechanisms," Renewable Energy, vol. 94, pp. 32-47, 2016.

[5] H. Mendonça and S. Martinez, "A resistance emulation approach to optimize the wave energy harvesting for a direct drive point absorber," IEEE Trans. Sustainable Energy, vol. 7, no. 1, pp. 3-11, 2016.

[6] E. Anderlini, D. I. M. Forehand, E. Bannon, and M. Abusara, "Reactive control of a wave energy converter using artificial neural networks," Inter. Journal of Marine Energy, vol. 19, pp. 207-220, 2017.

[7] M. K. Ochi, Ocean waves: The stochastic approach. USA: Cambridge ocean technology series 6, Cambridge Univ. Press, 1998.

[8] F. Fusco and J. V. Ringwood, "A simple and effective real-time controller for wave energy converters," IEEE Trans. Sustainable Energy, vol. 4, no. 1, pp. 21-30, 2013.

[9] P. B. Garcia-Rosa, G. Kulia, J. V. Ringwood, and M. Molinas, "Realtime passive control of wave energy converters using the Hilbert-Huang transform," in IFAC-PapersOnLine, vol. 50, no. 1 (Proc. of the 20th IFAC World Congress), Toulouse, France, 2017, pp. 14705-14 710.

[10] J. Hals, J. Falnes, and T. Moan, "A comparison of selected strategies for adaptive control of wave energy converters," J. of Offshore Mech. and Arctic Eng., vol. 133, no. 3, pp. 031 101-031 113, 2011.

[11] P. B. Garcia-Rosa, F. Lizarralde, and S. F. Estefen, "Optimization of the wave energy absorption in oscillating-body systems using extremum seeking approach," in Proc. of the IEEE American Control Conf. (ACC), Montréal, Canada, 2012, pp. 1011-1016.

[12] F. Fusco and J. V. Ringwood, "Hierarchical robust control of oscillating wave energy converters with uncertain dynamics," IEEE Trans. Sustainable Energy, vol. 5, no. 3, pp. 958-966, 2015.

[13] A. M. Cantarellas, D. Remon, and P. Rodríguez, "Adaptive vector control of wave energy converters," IEEE Trans. Ind. Electron., vol. 53, no. 3 , pp. 2382-2391, 2017.

[14] E. Tedeschi and M. Molinas, "Tunable control strategy for wave energy converters with limited power take-off rating," IEEE Trans. Ind. Electron., vol. 59, no. 10, pp. 3838-3846, 2012.

[15] A. H. Sakr, Y. H. Anis, and S. M. Metwalli, "System frequency tuning for heaving buoy wave energy converters," in Proc. of the IEEE Inter. Conf. on Advanced Intelligent Mechatronics (AIM), Busan, Korea, 2015, pp. 1367-1372.

[16] P. Rodríguez, A. Luna, R. S. Muñoz-Aguilar, I. Etxeberria-Otadui, R. Teodorescu, and F. Blaabjerg, "A stationary reference frame grid synchronization system for three-phase grid-connected power converters under adverse grid conditions," IEEE Trans. Power Electron., vol. 27, no. 1, pp. 99-112, 2011.

[17] B. Boashash, "Estimating and interpreting the instantaneous frequency of a signal - Part 1: Fundamentals." Proc. of the IEEE, vol. 80, no. 4, pp. 520-538, 1992.

[18] N. E. Huang, Z. Shen, S. R. Long, M. C. Wu, H. H. Shih, Q. Zheng, N.C. Yen, C. C. Tung, and H. H. Liu, "The empirical mode decomposition and the Hilbert spectrum for nonlinear and non-stationary time series analysis," Proc. Royal Society London, vol. 454, pp. 903-995, 1998.

[19] W. E. Cummins, "The impulse response function and ship motions," Schiffstechnik, vol. 47, no. 9, pp. 101-109, 1962.

[20] J. Falnes, Ocean Waves and Oscillating Systems: Linear Interaction including Wave-Energy Extraction. USA: Cambridge Univ. Press, 2002.

[21] K. Budal and J. Falnes, "A resonant point absorber of ocean-wave power," Nature, vol. 256, pp. 478-479, 1975.

[22] F. Fusco and J. V. Ringwood, "Short-term wave forecasting for real-time control of wave energy converters," IEEE Trans. Sustainable Energy, vol. 1, no. 2, pp. 99-106, 2010.

[23] A. C. Harvey, Forecasting, Structural Time Series Models and the Kalman Filter. Cambridge Univ. Press, 1989.

[24] B. F. La Scala and R. R. Bitmead, "Design of an extended Kalman filter tracker," IEEE Trans. Signal Process., vol. 44, no. 3, pp. 739-742, 1996.
[25] Z. Wu and N. E. Huang, "A study of the characteristics of white noise using the empirical mode decomposition method," Proc. Royal Society London, vol. 460, pp. 1597-1611, 2004.

[26] WAMIT, Inc., WAMIT User Manual Versions 6.4, 6.4PC and 6.3S, 6.3S$P C$, USA, 1998-2006.

[27] N. E. Huang, Z. Shen, and S. R. Lon, "A new view of nonlinear water waves: The Hilbert spectrum," Аnnu. Rev. Fluid Mech., vol. 31, pp. 417-457, 1999.

[28] Y.-Y. Hong and Y.-Q. Bao, "FPGA implementation for real-time empirical mode decomposition," IEEE Trans. Instrum. Meas., vol. 61, no. 12, pp. 3175-3184, 2012.

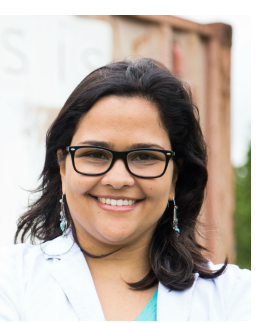

Paula B. Garcia-Rosa (S'13-M'13) received the B.Sc. degree in Electronic Engineering from the State University of Rio de Janeiro, Brazil, in 2004, and the M.Sc. and D.Sc. degrees in Electrical Engineering and Ocean Engineering, respectively, from the COPPE/Federal University of Rio de Janeiro, in 2008 and 2013. She was a Postdoctoral Researcher (2013-2015) with the Centre for Ocean Energy Research, Maynooth University, Ireland , and a Visiting Researcher (2016-2017) with the Dept. of Eng. Cybernetics, Norwegian Univ. of Science and Technology (NTNU). She is currently a Postdoctoral Researcher with the Dept. of Electric Power Eng. at NTNU. Her research interests include control of electromechanical systems, marine systems, renewable energy technologies and modeling, optimization and control of ocean energy systems.

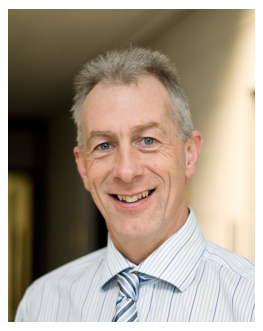

John Ringwood (M'87-SM'97) received the Diploma in Electrical Engineering from Dublin Institute of Technology and the PhD (in Control Systems) from Strathclyde University, Scotland, in 1981 and 1985 respectively. He is currently Professor of Electronic Engineering at Maynooth University Ireland and Director of the Centre for Ocean Energy Research there. John's research interests cover time series modelling, wave energy, control of plasma processes and biomedical engineering. He is a Chartered Engineer and a Fellow of Engineers Ireland.

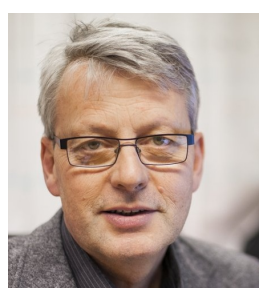

Olav B. Fosso (M'90-SM'06) is Professor at the Dept. of Electric Power Eng. of the Norwegian University of Science and Technology (NTNU). He has previously held positions as Scientific Advisor and Senior Research Scientist at SINTEF Energy Research, Head of the Dept. of Electric Power Eng. (2009-2013) and Director of NTNUs Strategic Thematic Area Energy (2014-2016). He has been Chairman of CIGRE SC C5 Electricity Markets and Regulation and Member of CIGRE Technical Committee (2008-2014), Chairman of the board of NOWITECH (2015-2017) and currently Board member of the Norwegian National Strategy Energy21. His research activities involve hydro scheduling, market integration of intermittent generation and signal analysis for study of power systems dynamics and stability.

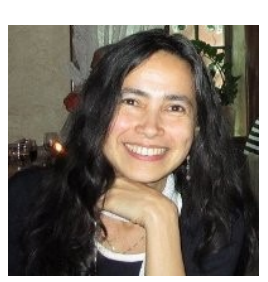

Marta Molinas (M'94) received the Diploma degree in Electromechanical Engineering from the National University of Asuncion, Paraguay, in 1992; the Master of Engineering degree from Ryukyu University, Japan, in 1997; and the Doctor of Engineering degree from the Tokyo Institute of Technology, Japan, in 2000. She was a Guest Researcher with the University of Padova, Italy, during 1998 and a JSPS Fellow in Japan from 2008 to 2009. She was a Postdoctoral Researcher (2004-2007) and Professor (2008-2014) at the Dept. of Electric Power Eng., Norwegian University of Science and Technology (NTNU). She is currently Professor at the Dept. of Eng. Cybernetics, NTNU. Her research interests include stability of power electronics systems, harmonics, instantaneous frequency, and non-stationary signals from the human and the machine. 